\title{
THE RATIONAL HOMOLOGY OF TORIC VARIETIES IS NOT A COMBINATORIAL INVARIANT
}

\author{
MARK MCCONNELL
}

(Communicated by Frederick R. Cohen)

\begin{abstract}
We prove that the rational homology Betti numbers of a toric variety with singularities are not necessarily determined by the combinatorial type of the fan which defines it; that is, the homology is not determined by the partially ordered set formed by the cones in the fan. We apply this result to the study of convex polytopes, giving examples of two combinatorially equivalent polytopes for which the associated toric varieties have different Betti numbers.
\end{abstract}

Our main result is that the rational homology Betti numbers of a toric variety with singularities are not necessarily determined by the combinatorial type of the fan which defines it; that is, the homology is not determined by the partially ordered set formed by the cones in the fan. This holds in all dimensions $n \geq 3$. The result is in contrast with the following facts:

(1) The rational homology Betti numbers of a nonsingular toric variety are determined by the combinatorial type of the fan. The rational cohomology ring of nonsingular toric varieties played a central role in the proof of McMullen's conjecture concerning the number of faces of simplicial convex polytopes [S1, BL].

(2) The intersection homology Betti numbers of a singular toric variety are determined by the combinatorial type of the fan. This fact provides information about general rational convex polytopes [S2].

We give an algorithm (1.2-1.3) for computing the Betti numbers of a complete toric variety of dimension three. We then give examples (1.4) of two combinatorially equivalent polyhedra for which the associated toric varieties have different Betti numbers. In $\S 2$ we prove the results in (1.2-1.3), and in $\S 3$ we conclude with a few remarks.

\section{Statement of Results}

(1.1) Let $\sigma$ denote any closed convex rational polyhedral cone in $\mathbf{R}^{n}$ which does not contain a line. A (complete) fan $\Sigma$ is a finite collection $\left\{\sigma_{\alpha}\right\}$ which

Received by the editors April 22, 1988.

1980 Mathematics Subject Classification (1985 Revision). Primary 52A25, 14L32.

Key words and phrases. Polytopes, toric varieties, $f$-vector. 
forms a rational polyhedral decomposition of $\mathbf{R}^{n}$. Any $\sigma_{\alpha} \in \Sigma$ is called a face of $\Sigma$. Faces of dimension $n$ are called chambers (denoted $\sigma_{j}$ ); faces of dimension one are called edges (denoted $\tau_{i}$ ). The number of faces of dimension $m$ is denoted $f_{m}$. The complete, normal toric variety $X=X_{\Sigma}$ is defined as in [TE1, §1.2 and D, §5].

Let $P \subset \mathbf{R}^{n}$ be a convex polytope of dimension $n$, all of whose vertices are rational, and which contains the origin in its interior. We construct a fan $\Sigma_{P}$ by taking all the cones whose vertices are at the origin and which are generated by the proper faces of $P$.

(1.2) Now let $\Sigma$ be a fan in dimension $n=3$. For each edge $\tau_{i}$, let $t_{i} \in \mathbf{Z}^{3} \subset$ $\mathbf{Q}^{3}$ be the unique vector $(\alpha, \beta, \gamma)$, with $\alpha, \beta, \gamma$ coprime, which generates $\tau_{i}$.

Consider the following diagram:

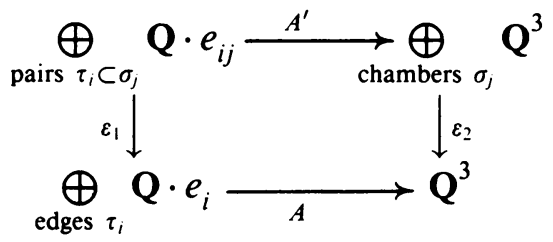

Here the $e_{i j}$ are a basis of an abstract vector space; the $e_{i}$ are interpreted similarly. $A$ is the obvious map $e_{i} \mapsto t_{i} . A^{\prime}$ uses the incidence relations in $\Sigma$ : for each pair $\tau_{i} \subset \sigma_{j}, A^{\prime}$ sends $e_{i j}$ to the vector $t_{i}$ in the $j$ th direct summand $\mathbf{Q}^{3}$. The vertical maps are natural projections: $\varepsilon_{1}$ sends $e_{i j}$ to $e_{i}$, and $\varepsilon_{2}$ is an augmentation map which adds up the vectors coming from the various summands which form its domain.

The vertical maps extend uniquely to kernels:

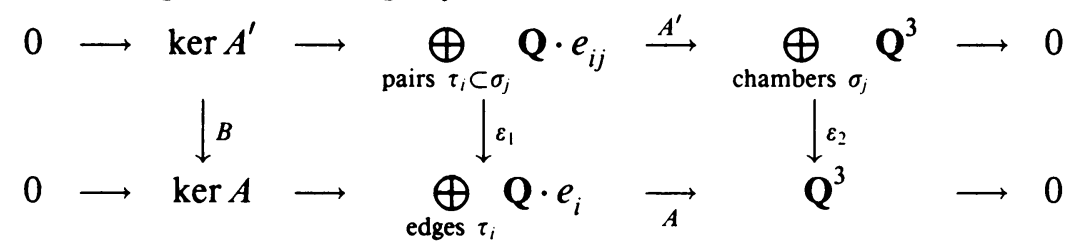

The number $b=\operatorname{rank} B$ is easily computed.

(1.3) Proposition. The Betti numbers $b_{k}=\operatorname{rank} H_{k}(X ; \mathbf{Q})$ of $X$ are

$$
1,0, f_{1}-b-3,3 f_{1}-f_{2}-b-6, f_{1}-3,0,1
$$

for $k=0, \ldots, 6$.

(1.4) Example. The standard rhombododecahedron $P_{1}$ is the convex hull in $\mathbf{R}^{3}$ of the fourteen points whose coordinates are the columns of the matrix

$$
\left(\begin{array}{rrrrrrrrrrrrr}
1 & 0 & 0 & -1 & 0 & 0 & \frac{1}{2} & \frac{1}{2} & \frac{1}{2} & \frac{1}{2} & -\frac{1}{2} & -\frac{1}{2} & -\frac{1}{2}-\frac{1}{2} \\
0 & 1 & 0 & 0 & -1 & 0 & \frac{1}{2} & \frac{1}{2} & -\frac{1}{2} & -\frac{1}{2} & \frac{1}{2} & \frac{1}{2} & -\frac{1}{2}-\frac{1}{2} \\
0 & 0 & 1 & 0 & 0 & -1 & \frac{1}{2} & -\frac{1}{2} & \frac{1}{2} & -\frac{1}{2} & \frac{1}{2} & -\frac{1}{2} & \frac{1}{2}-\frac{1}{2}
\end{array}\right)
$$


Another rhombododecahedron $P_{2}$ is the convex hull of the fourteen points whose coordinates are the columns of

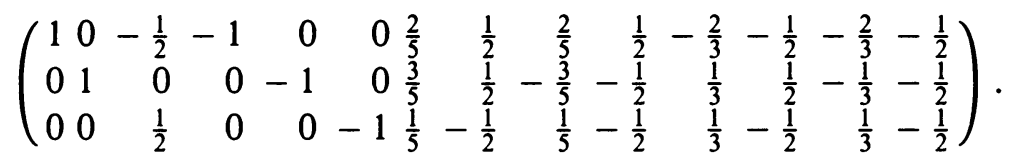

Both of these polyhedra have twelve quadrilateral faces and twenty-four edges, arranged as in the following Schlegel diagram (the numbers on the graph refer to the columns of (1.4.1), (1.4.2)):

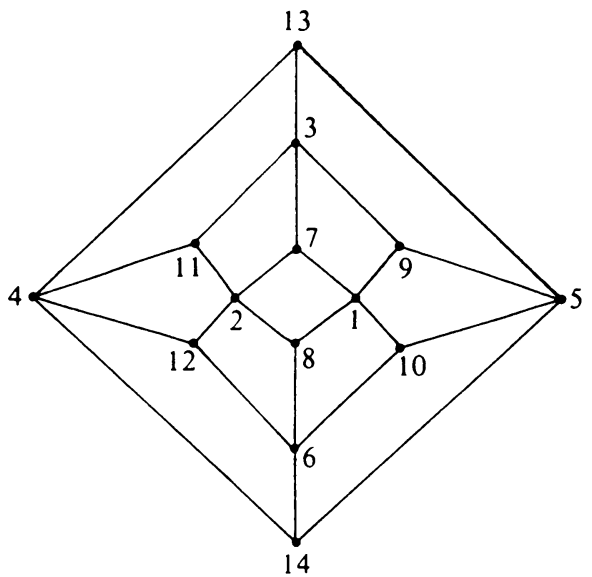

Thus the fans $\Sigma_{P_{1}}, \Sigma_{P_{2}}$ are equivalent as partially ordered sets. But by the algorithm of (1.2), the first fan yields a toric variety with Betti numbers

$$
1,0,2,3,11,0,1 \text {, }
$$

while the second yields a toric variety with Betti numbers

$$
1,0,1,2,11,0,1 \text {. }
$$

\section{Proof of Proposition 1.3}

Remark. In this paper, all homology groups have coefficients in $\mathbf{Q}$.

\subsection{Lemma. The Betti numbers}

$$
b_{0}, b_{1}, b_{4}, b_{5}, b_{6}
$$

of $X$ are given by

$$
1,0, f_{1}-3,0,1 \text {; }
$$

moreover, $b_{3}-b_{2}=2 f_{1}-f_{2}-3$.

Proof. This follows directly from a spectral sequence for the cohomology of $X$ found in [D, Theorem 12.2], together with the results [D, 12.7.1, 12.9, 12.10]. 
Remark. To prove Proposition 1.3, it thus suffices to compute $b_{2}$. The rest of $\S 2$ is devoted to this problem.

(2.2) We recall that to each $\sigma \in \Sigma$ corresponds an affine open set $X_{\sigma} \subset X$ (denoted $X_{\check{\sigma}}$ in [D, §5]). If we let $X_{i}=\bigcup\left\{X_{\sigma} \mid \operatorname{codim} \sigma \geq i\right\}$, then $X$ is naturally stratified in such a way that $X_{i} \backslash X_{i+1}$ is the collection of strata of real dimension $2 i[\mathrm{D}, \S 5.7]$; that is,

$$
X_{i} \backslash X_{i+1}=\coprod_{\{\sigma \mid \operatorname{codim} \sigma=i\}} S_{\sigma}
$$

where $S_{\sigma}$ is the connected stratum $X_{\sigma} \backslash X_{i+1}$.

(2.3) $H_{*}(X ; \mathbf{Q})$ is computed by the spectral sequence arising from the filtration by complements of strata-that is, the filtration $X=X_{0} \supset X_{1} \supset X_{2} \supset X_{3} \supset$ $\varnothing$. (More precisely, we filter by complements of tubular neighborhoods of the strata, so that the complements are closed sets.) We have

$$
\begin{aligned}
E_{q+k, q}^{1} & =H_{k}\left(X_{q}, X_{q+1}\right) \\
& \cong \bigoplus_{\operatorname{codim} \sigma=q} H_{k}\left(X_{\sigma}, X_{\sigma} \backslash S_{\sigma}\right) \text { by excision } \\
& \Longrightarrow H_{k}(X) .
\end{aligned}
$$

The fact is that this $E^{1}$ looks like

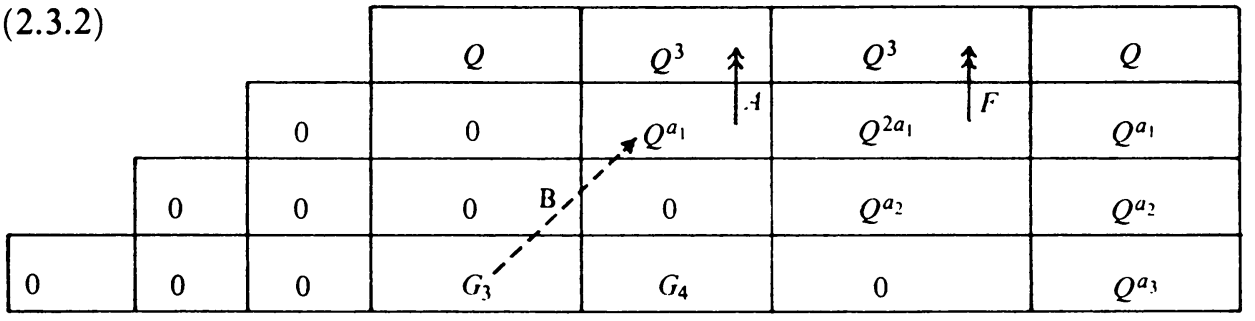

where $A$ and $F$ are $d_{1}$ differentials, and $B$ is a $d_{2}$ differential (to be used later).

Remarks. (1) We will not give a full proof that (2.3.2) is correct, since we will only need to use parts of the diagram. What we need will be proved in (2.4)-(2.7).

(2) The $E^{1}$ term is a combinatorial invariant. The $E^{2}$ term is an invariant except in $E_{5: 1}^{2}$, which is one of the boxes we will not need to study in this paper. (It can be shown that $E_{5,1}^{2} \cong I H_{4}^{\overline{0}}(X) \cong H^{2}(X)$, where $I H^{\overline{0}}$ denotes intersection homology in perversity $\overline{0}$.) The rank of $B$ is not an invariant, in spite of the fact that the dimensions of its source and target are invariants.

(2.4) Lemma. The top three rows $(q \geq 1)$ of (2.3.1) are as shown in (2.3.2). Proof. Choose $\sigma \in \Sigma$, with $\operatorname{codim} \sigma=q, q \geq 1$. The points of $S_{\sigma}$ are manifold points in $X$ when $q=2,3$ and are Q-homology manifold points 
when $q=1$ (as can be seen by the methods of [TE1, p. 19]). The methods of [AMRT, §1.1] show there is no monodromy in the links of these strata. The result follows easily, using the fact that $S_{\sigma} \cong\left(\mathbf{C}^{*}\right)^{q}$.

(2.5) Let $T^{m}$ denote the $m$-torus $S^{1} \times \cdots \times S^{1}$. We fix an identification of $X_{3} \cong\left(\mathbf{C}^{*}\right)^{3}$ with $\mathbf{R}^{3} \times T^{3}$, following [AMRT, §1.1] and [F]. Viewing $T^{3}$ as $\mathbf{R}^{3} / \mathbf{Z}^{3}$, we see that the line $\mathbf{R} \cdot t_{i} \subset \mathbf{R}^{3}$ induces a closed 1-cycle $\bar{t}_{i} \subset T^{3}$.

Lemma. The map $A$ in (2.3.2) is the same as $A$ in (1.2.1) for a suitable choice of basis.

Proof. In (2.3.2), domain $A=E_{4,2}^{1}=\bigoplus_{\text {edges } \tau_{i}} H_{2}\left(X_{\tau_{i}}, X_{\tau_{i}} \backslash S_{\tau_{i}}\right)$. As in (2.4), this is isomorphic to $\bigoplus_{\tau_{i}} H_{0}\left(S_{\tau_{i}}\right)$, which we write as $\bigoplus_{\tau_{i}} \mathbf{Q} \cdot e_{i}$ where $\left\{e_{i}\right\}$ is a basis of an abstract vector space.

By the methods of [AMRT, $\S 1.1$ or F], the link of $S_{\tau_{i}}$ at any $x \in S_{\tau_{i}}$ will lie in $X_{3}$ as a copy of $\bar{t}_{i} \subset T^{3}$. Since $t_{i}$ is a coprime integer vector, the coefficients of $\bar{t}_{i}$ in a standard basis of $H_{1}\left(T^{3}\right)$ are the components of $t_{i}$. Thus, in both (2.3.2) and (1.2.1), $A$ sends $e_{i}$ to $t_{i}$.

(2.6) Lemma. The map $F$ in (2.3.2) is surjective.

Proof. This follows exactly as in the smooth case (see [D, §12]).

(2.7) Lemma. The group $G_{3}$ in (2.3.2) can be identified as $\operatorname{ker} A^{\prime}$ in (1.2.1). Also, $E_{2,0}^{1}=0$.

Proof. The proof is an exercise in algebraic topology. The identification of $G_{3}$ with $\operatorname{ker} A^{\prime}$ follows as in (2.5).

(2.8) We can now complete the proof of Proposition 1.3. We have shown that, when the spectral sequence converges, all of $H_{2}(x)$ will live in the box $E_{4,2}^{\infty}$; it will be the cokernel of a $d_{2}$ differential $B: G_{3} \rightarrow \operatorname{ker} A$. But $G_{3} \cong \operatorname{ker} A^{\prime}$, and one checks (referring to (2.5) and (2.7)) that this $B$ is the same as the naturally induced map $B$ of (1.2.1). Thus $b_{2}=\left(f_{1}-3\right)-b$.

\section{REMARKS}

(3.1) Fix a class $\mathscr{S}$ of fans $\Sigma$ of a given combinatorial type. Since the $b_{k}$ are determined by a rank condition, there is a Zariski open set (i.e., complement of a proper subvariety) in $\mathscr{S}$ where the fans have a certain list of Betti numbers (the generic values of the Betti numbers). There are degeneracy loci where the fans yield other lists. This holds in all dimensions $n \geq 3$. (3.2) Fix $\Sigma$ of dimension $n=3$. Let $\theta$ be a collection of faces $\theta \in \Sigma$ of dimension two such that, for every $\tau_{i} \in \Sigma$, there is a chamber $\sigma_{j(i)}$ and a face $\theta_{k(i)} \in \Theta$ such that $\tau_{i} \subset \sigma_{j(i)}$ and $\theta_{k(i)} \subset \sigma_{j(i)}$. This depends only on the poset structure of $\Sigma$. Let $N_{\Theta}$ be the minimum, over all possible such collections $\Theta$,

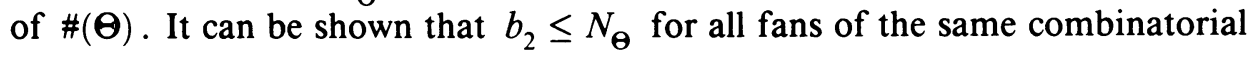


type as $\Sigma$. We do not know when this bound is sharp, or whether every value of $b_{2}$ between the generic value and $N_{\Theta}$ is attained as $\Sigma$ varies within a given combinatorial type.

\section{ACKNOWLEDGMENT}

I would like to thank Joe Harris, Bill Fulton, Jonathan Fine, and Mark Goresky for helpful conversations, and the referee for helpful comments. My special thanks go to Bob MacPherson for introducing me to this problem, and for his advice and support.

\section{REFERENCES}

[AMRT] A. Ash, D. Mumford, M. Rapoport, Y. Tai, Smooth compactifications of locally symmetric varieties, in Lie Groups: History, Frontiers, and Applications, vol. 4, Math. Sci. Press, 1975.

[BL] L. J. Billera, C. W. Lee, Sufficiency of McMullen's conditions for $f$-vectors of simplicial polytopes, J. Combin. Theory Ser. A 31, no. 3 (1981), 237-255.

[D] V. I. Danilov, The geometry of toric varieties, Russian Math. Surveys 33:2 (1978), 97-154.

[F] J. Fine, Geometric progressions, convex polytopes, and torus embeddings (preprint).

[S1] R. Stanley, The number of faces of a simplicial convex polytope, Adv. in Math. 35 (1980), 236-238.

[S2] _ Generalized H-vectors, intersection cohomology of toric varieties, and related results, Commutative Algebra and Combinatorics, Adv. Stud. Pure Math., vol. 11, ed. M. Nagata and H. Matsumura, pub. Kinokuniya, Tokyo and New York, (1987), pp. 187-213.

[TE1] G. Kempf, F. Knudsen, D. Mumford, B. Saint-Donat, Toroidal embeddings I, Lecture Notes in Math., vol. 339, Springer-Verlag, Berlin and New York, 1973, 209 pp.

Department of Mathematics, Harvard University, Cambridge, Massachusetts 02138 